\title{
MOTIVACIONES PARA ELEGIR LA PROFESIÓN DOCENTE EN ESTUDIANTES QUE INGRESAN A LA EDUCACIÓN SUPERIOR
}

\section{MOTIVATIONS FOR CHOOSING THE TEACHING PROFESSION IN STUDENTS ENTERING HIGHER EDUCATION}

\author{
Raúl Prada Núñez' \\ César Augusto Hernández Suárez² \\ William Rodrigo Avendaño Castro ${ }^{3}$ \\ UFPS
}

\section{RESUMEN}

Diversas investigaciones han resaltado la importancia de la labor docente en el éxito académico de los estudiantes. Un aspecto

$1 \quad$ Magíster en Ingeniería de Análisis de Datos, Mejora de Procesos y Toma de Decisiones por la Universidad Politécnica de Valencia (España). Docente investigador de la Universidad Francisco de Paula Santander. Correo electrónico: raulprada@ufps.edu.co. Orcid: 0000-0001-61451786.

$2 \quad$ Magister en Educación Matemática por la Universidad Nacional Experimental del Táchira (Venezuela). Docente investigador de la Universidad Francisco de Paula Santander. Correo electrónico: cesaraugusto@ufps.edu.co. Orcid: 0000-0002-7974-5560.

3 Doctor en Ciencias Sociales y Humanas por la Pontificia Universidad Javeriana. Docente investigador de la Universidad Francisco de Paula Santander. Correo electrónico: williamavendano@ufps.edu.co. Orcid: 0000-00027510-8222. importante dentro de este escenario de múltiples factores incidentes asociados con el docente sería entre muchos otros, las características de su práctica pedagógica, los recursos que utiliza y no menos importante, las motivaciones que han tenido y que los llevó a ingresar a un programa académico de formación docente. Es en este sentido que se orienta esta investigación, a través de la cual se pretende identificar los intereses y motivaciones que han llevado a un grupo de 119 graduados de la educación media a elegir un programa de Licenciatura como su proyecto de vida y laboral. Este programa se oferta en una universidad pública desde hace tres semestres y ha demostrado tener alta 
pertinencia en el entorno social dada el alto volumen de solicitudes de ingreso que se han presentado en este tiempo. Para tal fin se diseñó un cuestionario ad hoc que se compone de cuatro secciones en dónde se evalúan las motivaciones generales, las motivaciones intrínsecas, las motivaciones trascendentes y las motivaciones extrínsecas. En todas las cuatro categorías de análisis se considera una escala Likert con cinco niveles de aceptación. Los resultados permiten identificar como principal motivación el gusto al trabajar con niños acompañado de su vocación docente, pero hay ausencia de competencias pedagógicas que se espera se solventen en la medida que avanza en su proceso de formación profesional.

\section{PALABRAS CLAVE}

Formación de docentes, vocación docente, intereses y afinidades, educación superior.

\section{ABSTRACT}

Several research studies have highlighted the importance of the teacher's work in the academic success of students. An important aspect within this scenario of multiple incident factors associated with teachers would be, among many others, the characteristics of their pedagogical practice, the resources they use and, not least, the motivations they have had and that led them to enter an academic teacher training program. It is in this sense that this research is oriented, through which it is intended to identify the interests and motivations that have led a group of 119 high school graduates to choose a Bachelor's degree program as their life and work project. This program has been offered in a public university for three semesters and has proven to be highly relevant in the social environment given the high volume of applications for admission that have been presented during this time. For this purpose, an ad hoc questionnaire was designed with four sections where general motivations, intrinsic motivations, transcendent motivations and extrinsic motivations are evaluated. In all four categories of analysis, a Likert scale with five levels of acceptance is considered. The results allow us to identify as the main motivation the pleasure of working with children together with their teaching vocation, but they lack pedagogical competencies that are expected to be solved as they advance in their professional training process.

\section{KEYWORDS}

Teacher training, teaching vocation, interests and affinities, higher education.

\section{INTRODUCCIÓN}

La motivación que tienen las personas para elegir programas profesionales es un campo que se ha estudiado de manera amplia en todos los escenarios del mundo. Diversas investigaciones han determinado que las personas se inclinan por las carreras profesionales por gusto o por interés y no necesariamente por las salidas laborales que ofrecen los diferentes grados universitarios (Rodríguez et al., 2019). Otros estudios (Games y Marrero, 2003, Troncoso, Garay y Sanhueza, 2016) revelan que la principal motivación que tienen algunos estudiantes para escoger su carrera es el prestigio que obtienen al estudiar un programa como medicina o derecho, por ejemplo.

Por su parte, Centurión (2014) afirma que los padres y los profesores también influyen en la toma de decisión por parte de los alumnos para escoger una carrera, asi como la disponibilidad de la carrera deseada, la adquisición de becas de estudio y la cercanía de la universidad con la casa.

De otro modo, el término motivación también se relaciona con la orientación vocacional, la cual según Vidal y Fernández (2009) se refiere a ser entendida como un proceso que dé ayuda a la elección de una profesión, la preparación para ella, el acceso al ejercicio de la misma y 
la evolución y progreso posterior. Este proceso tiene como objetivo despertar los intereses vocacionales que el individuo requiere, el conocimiento de sí mismo, de las ofertas capacitantes y académicas, de los planes y programas de estudio, de las propuestas de trabajo, de las competencias que debe desarrollar para alcanzar un buen desempeño en esas propuestas, lo cual le permitirá tomar las decisiones que considere de acuerdo a sus capacidades y aptitudes para ubicarse en el contexto social-laboral (p.87).

Otros autores como Mendoza y Rodríguez (2008) consideran que la orientación vocacional a modo general intenta descubrir el potencial de cada sujeto y ver que cada uno tenga su oportunidad para desarrollar ese potencial al máximo, en lo que mejor pueda ofrecer a sí mismo y al mundo. Se plantea como un proceso o conjunto de acciones para ayudar a otros en la solución de situaciones críticas y conflictivas o en la satisfacción de necesidades para el logro de un estado de bienestar.

Es importante analizar ahora la relevancia de la vocación docente, teniendo en cuenta que los profesores son los guías en el aprendizaje de los estudiantes y el amor y el gusto por esta profesión es el detonante de una buena educación. En este sentido una gama importante de de investigaciones se ha centrado en indagar sobre la pasión por enseñar (Day, 2006), el compromiso del maestro (Fuentealba, 2014) y el liderazgo profesional (Vernal et al., 2013).

Sin duda, las características descritas en el párrafo anterior se deben tener en cuenta antes de ingresar a un programa de educación superior de formación de maestros y tener muy claras las motivaciones para no llegar a ser lo que algunos llaman "profesor por accidente" o "profesor por necesidad" (Sánchez, 2002). En este contexto se han realizado diversos estudios relacionados con la motivación de los estudiantes para escoger programas de formación de maestros con importantes hallazgos que revelan que las motivaciones de tipo altruistas e intrínsecas son las más valoradas por los estudiantes y las motivaciones extrínsecas, relacionadas con el salario y la conciliación, por ejemplo, tienen una valoración menor, con la excepción del factor relacionado con la seguridad del trabajo. La satisfacción de la elección es elevada pese al bajo salario, la falta de estatus social y una alta disuasión social (Said, Gratacós y Cobos, 2017, p.38).

Del mismo modo, otros elementos que motivan de manera significativa a los estudiantes a inclinarse con la carrera docente se relacionan con el salario, las vacaciones, el horario, pero también otros más subjetivos como la vocación, ilusiones o satisfacción personal (Sanchez, 2009).

Por estos elementos descritos se hace necesario realizar un diagnóstico sobre la motivación que tuvieron los estudiantes para ingresar al programa de licenciatura en educación infantil en la universidad objeto de estudio y de esta manera generar espacios de reflexión y planes de mejora dentro de la administración del programa con esta información anticipada que se obtuvo en esta investigación.

\section{METODOLOGÍA}

Para la recolección de los datos que permitieran dar respuesta al objetivo propuesto en esta investigación se procedió inicialmente a invitar a la totalidad de estudiantes matriculados en el primer semestre del 2021 en un programa de formación de maestros para el nivel de educación preescolar. Este programa a la fecha está ofertando los tres primeros semestres de su propuesta curricular de nueve semestres dado que recibió registro calificado en septiembre del 2019. Es un programa pertinente en la región dado su alto nivel de demanda observado en el tiempo de funcionamiento, por ende, se adopta 
un enfoque cuantitativo a nivel descriptivo y con diseño de campo.

En esta investigación no se aplica proceso de muestreo pues se pretende adelantar un estudio censal. Se diseñó un cuestionario ad hoc a partir de la revisión de antecedentes, el cual ha sido avalado por un panel de expertos.

El cuestionario se compone de varias secciones: a) Información general orientada a determinar aspectos característicos a nivel demográfico de los encuestados; b) Motivaciones generales con lo que se pretende realizar una aproximación a ciertas generalidades que podrían ser consideradas al momento de seleccionar el programa de formación de maestros; c) Motivaciones intrínsecas conceptualizada como una "propensión innata" (Ryan y Deci, 2000, p.71) del individuo y puede identificarse a través de la presencia de ciertas acciones o actitudes que no basan en una recompensa externa, al reflejar un interés desinteresado hacia la profesión docente; d) Motivaciones trascendentes que hacen referencia al interés de utilizar los conocimientos generados en la formación docente desde una perspectiva de contribución social, donde el trabajo docente es importante y valioso por sus implicaciones para los otros (Kyriacou y Coulthard, 2000; LópezJurado y Gratacós, 2013); e) Motivaciones extrínsecas las cuales aluden a las situaciones que no son inherentes al individuo o a la práctica docente, sino que se presentan de manera exógena y que tienen un impacto en la elección (Kyriacou y Coulthard, 2000).

En todos los ítems se han presentado opciones de respuesta cerradas y en el caso de la exploración de las motivaciones, se ha recurrido al uso de una escala Likert con cinco niveles de respuesta: dos niveles de rechazo, un nivel indiferente y dos niveles de aceptación. Una vez aprobado el cuestionario, se procedió a generar un Google Form que posteriormente fue compartido al grupo de estudiantes matriculados en el programa objeto de estudio, durante la semana de matrícula, inclusiones y cancelaciones de asignaturas. Los datos recolectados fueron descargados en un archivo de Excel para posteriormente ser exportados al SPSS v25 y darles un tratamiento descriptivo.

\section{RESULTADOS Y DISCUSIÓN}

Perfil Demográfico. Por medio de la Tabla 1 se reportan las características demográficas del grupo de estudiantes encuestados. Se pudo identificar el predominio del género femenino, con edades entre 16 y 21 años, provenientes principalmente de instituciones educativas cuyos énfasis de formación en la media técnica no son pedagógicos. En cuanto a las condiciones económicas, se identificó que aproximadamente el $91.6 \%$ corresponden a los estratos uno y dos. Finalmente se identificó que tres de cada cuatro estudiantes que han ingresado al programa académico, ingresan por primera vez al sistema de educación superior. 
Tabla 1. Perfil demográfico de los encuestados

\begin{tabular}{|c|c|c|}
\hline Característica & Opciones de respuesta & Porcentaje \\
\hline \multirow{3}{*}{ Género } & Femenino & $96.6 \%$ \\
\hline & Masculino & $3.4 \%$ \\
\hline & Total & $100.0 \%$ \\
\hline \multirow{4}{*}{ Edad } & Entre 16 y 18 años & $42.9 \%$ \\
\hline & Entre 19 y 21 años & $54.7 \%$ \\
\hline & Entre 22 y 24 años & $2.5 \%$ \\
\hline & Total & $100.0 \%$ \\
\hline \multirow{4}{*}{$\begin{array}{c}\text { Énfasis en la Educación Media } \\
\text { Técnica }\end{array}$} & Bachillerato Académico & $55.5 \%$ \\
\hline & Normalista & $8.4 \%$ \\
\hline & Bachillerato Técnico & $36.1 \%$ \\
\hline & Total & $100.0 \%$ \\
\hline \multirow{4}{*}{$\begin{array}{l}\text { Estrato socioeconómico del } \\
\text { domicilio en dónde vive }\end{array}$} & Estrato 1 & $42.0 \%$ \\
\hline & Estrato 2 & $49.6 \%$ \\
\hline & Estrato 3 & $8.4 \%$ \\
\hline & Total & $100.0 \%$ \\
\hline \multirow{3}{*}{$\begin{array}{c}\text { ¿Estudió otro programa antes de } \\
\text { ingresar a la licenciatura? }\end{array}$} & $\mathrm{Si}$ & $24.4 \%$ \\
\hline & No & $75.6 \%$ \\
\hline & Total & $100.0 \%$ \\
\hline
\end{tabular}

Fuente. Elaboración propia

Con respecto al género, es importante señalar que estos resultados, independiente de la muestra tomada para el estudio, se pueden constrastar con diversas investigaciones que señalan que las mujeres tienen una tendencia mayor a estudiar el programa de licenciatura en educación infantil que los hombres por tener mayor motivación y sensibilidad por la primera infancia (Soza, Talavera y Gutierrez, 2017).

\section{MOTIVACIONES GENERALES.}

Para iniciar el proceso de caracterización de las motivaciones que han llevado a los estudiantes de este programa a seleccionarlo como proyecto de vida, se determinó que el $59.7 \%$ de ellos manifestaron el gusto que experimentan al trabajar con niños, seguido del $27.7 \%$ quienes aseguran que su principal motivación se deriva de que consideran poseen vocación docente acompañado del dominio sobre ciertas habilidades pedagógicas. El porcentaje restante, corresponde la estabilidad laboral que ofrece la profesión docente y la influencia de la familia en esta elección. 


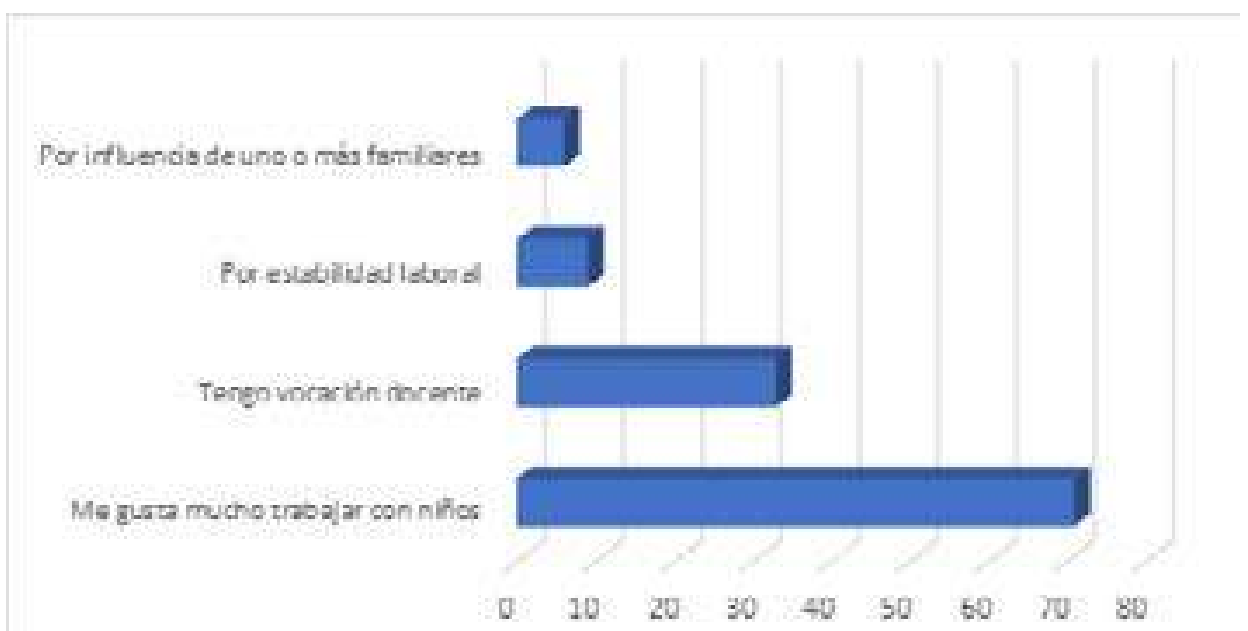

Figura 1. Frecuencia sobre las razones que influyeron para la elección del programa de Licenciatura en Educación Infantil

Los resultados que se muestran en la tabla anterior se pueden contrastar con estudios como el de Zapata y Ceballos (2000) quienes afirman que el gusto para trabajar con los niños y niñas es una actitud vocacionalidad predominante, pues un educador o educadora, además de gustarle, de querer a los niños y niñas y de sentirse identificado o identificada con sus funciones, se caracteriza por su profesionalismo y pasión por lo que se hace.

Explorando las motivaciones generales, se les presentó a los estudiantes una lista de aspectos básicos para que a partir de ellos auto reflexionaran sobre su nivel de competencias que poseían sobre la profesión docente (GarcíaQuintero \& Villamizar-Suárez, 2017; VillamizarAcevedo, Lozano-León \& Sierra-Garavito, 2017). Estos resultados se visualizan en la Tabla 2 a partir de la cual se resalta como fortaleza que aproximadamente uno de cada dos estudiantes que se han matriculado en el programa, se consideran competentes en cuanto a tener vocación docente puesto que poseen cualidades especiales para la enseñanza, reconociendo que es una actividad que demanda de constante actualización. En promedio el $40.9 \%$ se muestran inseguros en cuanto el nivel de dominio de las competencias docentes mencionadas, mientras que el $7.8 \%$ en general aseguran desconocer las funciones docentes, debido a que nunca han tenido contacto con el campo laboral y por ende sus habilidades pedagógicas no son las adecuadas. 
Tabla 2. Exploración de competencias básicas que poseen los estudiantes sobre los aspectos asociados con el desempeño docente

\begin{tabular}{|c|c|c|c|}
\hline \multirow{2}{*}{ Aspectos analizados } & \multicolumn{3}{|c|}{ Nivel de desempeño } \\
\hline & Ninguno & Parcial & Total \\
\hline $\begin{array}{l}\text { ¿Conoce sobre las diversas funciones } \\
\text { docentes? }\end{array}$ & $13.4 \%$ & $65.5 \%$ & $21.0 \%$ \\
\hline $\begin{array}{l}\text { ¿Considera que posee competencias para la } \\
\text { docencia? }\end{array}$ & $1.7 \%$ & $55.5 \%$ & $42.9 \%$ \\
\hline $\begin{array}{l}\text { ¿Ha tenido contacto con su futuro campo de } \\
\text { acción laboral? }\end{array}$ & $24.4 \%$ & $35.3 \%$ & $40.3 \%$ \\
\hline $\begin{array}{l}\text { ¿Considera que sabe transmitir los } \\
\text { conocimientos propios de su futuro laboral? }\end{array}$ & $10.1 \%$ & $47.1 \%$ & $42.9 \%$ \\
\hline $\begin{array}{l}\text { ¿Sabe cómo mantener el orden y controlar el } \\
\text { proceso de enseñanza? }\end{array}$ & $5.0 \%$ & $46.2 \%$ & $48.7 \%$ \\
\hline $\begin{array}{l}\text { ¿Dispone de valores adecuados y cualidades } \\
\text { especiales para la enseñanza? }\end{array}$ & $3.4 \%$ & $27.7 \%$ & $68.9 \%$ \\
\hline ¿Considera que tiene vocación docente? & $0.8 \%$ & $32.8 \%$ & $66.4 \%$ \\
\hline $\begin{array}{l}\text { ¿Considera que la labor docente demanda de } \\
\text { un proceso de constante actualización? }\end{array}$ & $3.4 \%$ & $16.8 \%$ & $79.8 \%$ \\
\hline Porcentaje Promedio & $7.8 \%$ & $40.9 \%$ & $51.3 \%$ \\
\hline
\end{tabular}

Fuente. Elaboración propia

Con respecto a este aspecto Zapata y Ceballos (2020) afirman que el educador infatil debe tener la habilidad y las competencias para ser consecuente con las demandas y características de los contextos en coherencia con la Política de Infancia que se tenga, centrado en el reconocimiento del niño y la niña como sujetos de derecho, en el enfoque de atención integral (educación, salud y protección) y en el acompañamiento afectivo caracterizado por una clara intencionalidad pedagógica, que rompa con el esquema de escolarización temprana y posibilite el desarrollo no sólo de las capacidades cognitivas, comunicativas y afectivas sino el desarrollo de habilidades para la vida, a través de la lúdica y el juego, haciendo partícipes de dicho proceso a la familia y a la sociedad como agentes educativos corresponsables y garantes de un verdadero desarrollo integral (p.1070).

\section{MOTIVACIONES INTRÍNSECAS PARA LA ELECCIÓN DE LA FORMACIÓN COMO DOCENTE.}

Como se mencionó anteriormente, en esta categoría de análisis se pretende caracterizar la presencia de ciertas actitudes que se derivan del interés desinteresado de recompensa externa tras la profesión docente. Dado que la escala Likert que se aplicó consideraba dos niveles de rechazo y dos de aceptación, para facilitar la interpretación estos dos niveles se agruparon en una categoría, por lo que en la Tabla 3 se muestran tres niveles de aceptación. 
Tabla 3. Exploración de motivaciones intrínsecas hacia la profesión docente

\begin{tabular}{lccc}
\hline \multicolumn{1}{c}{ Aspectos analizados } & \multicolumn{3}{c}{ Nivel de Aceptación } \\
\cline { 2 - 4 } & $\begin{array}{l}\text { En } \\
\text { desacuerdo }\end{array}$ & Indiferente & De acuerdo \\
\hline $\begin{array}{l}\text { Por el deseo de aportar en la construcción de } \\
\text { un mejor país. }\end{array}$ & $3.3 \%$ & $1.7 \%$ & $95.0 \%$ \\
\hline $\begin{array}{l}\text { Con mi trabajo, puedo aportar a la formación de } \\
\text { los niños de la región. }\end{array}$ & $4.2 \%$ & $3.4 \%$ & $92.5 \%$ \\
\hline $\begin{array}{l}\text { Me gusta compartir tiempo con los niños. } \\
\text { Se me facilita despertar el interés de los niños. }\end{array}$ & $2.5 \%$ & $2.5 \%$ & $95.0 \%$ \\
\hline $\begin{array}{l}\text { Desde que era niño(a) me ha gustado la } \\
\text { actividad docente. }\end{array}$ & $10.1 \%$ & $20.2 \%$ & $6.7 \%$ \\
\hline $\begin{array}{l}\text { En mis juegos infantiles siempre asumía el rol } \\
\text { de docente. }\end{array}$ & $9.2 \%$ & $19.3 \%$ & $71.5 \%$ \\
\hline $\begin{array}{l}\text { El deseo de enseñar se ha mantenido constante } \\
\text { a través del tiempo. }\end{array}$ & $8.4 \%$ & $10.9 \%$ & $80.6 \%$ \\
\hline \multicolumn{1}{c}{$\quad$ Porcentaje Promedio } & $\mathbf{5 . 7 \%}$ & $\mathbf{9 . 2} \%$ & $\mathbf{8 5 . 0 \%}$ \\
\hline
\end{tabular}

Fuente. Elaboración propia

Como se puede apreciar en la tabla anterior, en promedio el $85.0 \%$ de los estudiantes encuestados están de acuerdo con los diversos aspectos evaluados en esta categoría, destacándose como fortalezas el reconocimiento del efecto que tendría en la región o el país el buen desempeño de su rol docente, así como la afinidad que manifiestan sobre el compartir tiempo con los niños. Así mismo, en promedio el $5.7 \%$ reconocen que la actual elección como futuros docentes no ha sido algo que venían desde tiempo atrás.

\section{MOTIVACIONES TRASCENDENTES PARA LA ELECCIÓN DE LA FORMACIÓN COMO DOCENTE.}

En esta categoría de análisis se busca describir las expectativas sociales que poseen los estudiantes sobre su futuro desempeño profesional. La escala valorativa sufrió el mismo ajuste que en la categoría anterior. A través de la Tabla 4 se puede evidenciar la fuerte convicción que poseen los estudiantes de este programa académico en lo que respecta a su aporte desde su rol laboral, a la construcción de sociedad a partir de un proceso de formación integral de los niños. A pesar de que más del $80 \%$ de estos estudiantes no han tenido contacto con su campo laboral, si identifican como una debilidad para el ejercicio de su profesión, la falta de reconocimiento social, así como el abandono de la comunidad para garantizar la efectividad del proceso educativo, es decir, que los padres y demás miembros de la sociedad asumen que el proceso de formación es sólo responsabilidad de los docentes. 
Tabla 4. Exploración de motivaciones trascendentes hacia la profesión docente

\begin{tabular}{lccc}
\hline \multicolumn{1}{c}{ Aspectos analizados } & \multicolumn{3}{c}{ Nivel de Aceptación } \\
\cline { 2 - 4 } & $\begin{array}{c}\text { En } \\
\text { desacuerdo }\end{array}$ & Indiferente & De acuerdo \\
\hline $\begin{array}{l}\text { Desde mi rol como docente, puedo aportar a la } \\
\text { formación académica de los niños. }\end{array}$ & $2.5 \%$ & $0.0 \%$ & $97.5 \%$ \\
\hline $\begin{array}{l}\text { Desde mi rol como docente, puedo aportar a la } \\
\text { formación en valores de los niños. }\end{array}$ & $3.3 \%$ & $0.0 \%$ & $96.7 \%$ \\
\hline $\begin{array}{l}\text { Desde mi rol como docente, puedo aportar a la } \\
\text { construcción de una mejor sociedad. }\end{array}$ & $2.5 \%$ & $0.8 \%$ & $96.7 \%$ \\
\hline $\begin{array}{l}\text { La labor docente es un medio para ayudar a las } \\
\text { personas a progresar y a obtener mejor calidad } \\
\text { de vida. }\end{array}$ & $3.4 \%$ & $3.4 \%$ & $93.2 \%$ \\
\hline $\begin{array}{l}\text { Como docente puedo ser agente generador de } \\
\text { cambio en la comunidad donde labore. }\end{array}$ & $2.5 \%$ & $0.8 \%$ & $96.7 \%$ \\
\hline $\begin{array}{l}\text { La actividad docente tiene gran reconocimiento } \\
\text { social. }\end{array}$ & $16.8 \%$ & $20.2 \%$ & $63.0 \%$ \\
\hline $\begin{array}{l}\text { Considera que falta más apoyo de la comunidad } \\
\text { para que los docentes puedan ejercer mejor su } \\
\text { labor. }\end{array}$ & $10.1 \%$ & $4.2 \%$ & $85.7 \%$ \\
\hline \multicolumn{1}{c}{ Porcentaje Promedio } & $\mathbf{5 . 9 \%}$ & $\mathbf{4 . 2 \%}$ & $\mathbf{8 9 . 9 \%}$ \\
\hline
\end{tabular}

Fuente. Elaboración propia

\section{MOTIVACIONES EXTRÍNSECAS PARA LA ELECCIÓN DE LA FORMACIÓN COMO DOCENTE.}

En esta categoría se analizan los diversos aspectos externos que influyen de forma directa en el interés que muestren los estudiantes en la elección de un programa académico. La escala valorativa sufrió el mismo ajuste que en la categoría anterior.

Tabla 5. Exploración de motivaciones extrínsecas hacia la profesión docente

\begin{tabular}{|c|c|c|c|}
\hline \multirow[b]{2}{*}{ Aspectos analizados } & \multicolumn{3}{|c|}{ Nivel de Aceptación } \\
\hline & $\begin{array}{c}\text { En } \\
\text { desacuerdo }\end{array}$ & Indiferente & De acuerdo \\
\hline $\begin{array}{l}\text { El salario que recibe el docente es adecuado } \\
\text { para la función social que desarrolla. }\end{array}$ & $63.0 \%$ & $17.6 \%$ & $19.4 \%$ \\
\hline $\begin{array}{l}\text { Son limitadas las oportunidades que ofrece el } \\
\text { sistema educativo para aumentar el salario. }\end{array}$ & $15.1 \%$ & $16.8 \%$ & $68.1 \%$ \\
\hline $\begin{array}{l}\text { En mi casa me presionaron para elegir la } \\
\text { profesión docente. }\end{array}$ & $89.9 \%$ & $5.9 \%$ & $4.2 \%$ \\
\hline $\begin{array}{l}\text { Sentí presiones sociales para elegir la profesión } \\
\text { docente. }\end{array}$ & $94.1 \%$ & $1.7 \%$ & $4.2 \%$ \\
\hline La profesión docente ofrece estabilidad laboral. & $12.6 \%$ & $12.6 \%$ & $74.8 \%$ \\
\hline $\begin{array}{l}\text { La actividad docente siempre es necesaria, por } \\
\text { ello siempre hay opciones laborales. }\end{array}$ & $13.5 \%$ & $10.9 \%$ & $75.6 \%$ \\
\hline
\end{tabular}


Elegí el programa de Licenciatura en Educación Infantil porque no pude ingresar al programa $80.6 \%$ $5.0 \%$ $14.4 \%$ que realmente deseaba estudiar.

\begin{tabular}{cccc}
\hline $\begin{array}{l}\text { Deseo cambiarme a otro programa académico } \\
\text { porque descubrí que la docencia no es lo mío. }\end{array}$ & $95.8 \%$ & $1.7 \%$ & $2.5 \%$ \\
\hline Porcentaje Promedio & $\mathbf{5 8 . 1 \%}$ & $\mathbf{9 . 0} \%$ & $\mathbf{3 2 . 9} \%$ \\
\hline
\end{tabular}

Fuente. Elaboración propia

A través de la Tabla 5 se puede evidenciar el rechazo en más de la mitad de los estudiantes del programa ante las situaciones consideradas como motivaciones extrínsecas para la elección de la profesión docente, resaltando como aspectos destacados que nunca recibieron presiones de la familia o de su contexto para elegir este programa de formación a pesar de la influencias que ellos podrían tener en la elección (Méndez-Omaña \& Jaimes-Contreras, 2018), reconocen que están en el programa por vocación e interés propio el cual se manifiesta en su rechazo a cambiarse de programa, a pesar de considerar que económicamente la actividad docente no es bien remunerada puesto que son escasas las oportunidades de mejorar el salario, pero aún así a pesar de las dificultades están totalmente convencidos que siempre serán necesarios los docentes por lo que en cierta forma, tienen asegurada su estabilidad laboral.

Con relación a este aspecto hay estudios relevantes que muestran la inconformidad y algunas veces la falta de valoración de los profesionales de la educación. Estos determinantes de la desmotivación de los docentes se centran según Sanchez (2009) en la falta de oportunidades para ascender en el contexto educativo, poco apoyo de las instituciones para continuar con la preparación académica y una baja remuneración salarial y reconocimiento social.

A nivel general y estableciendo un comparativo entre las diversas categorías analizadas en lo concerniente a la caracterización de las motivaciones que han llevado a los estudiantes del programa de Licenciatura en Educación Infantil a seleccionar este programa como su proyecto de vida, se pudo determinar que las motivaciones trascendentes son las principales consideraciones que han influido en la elección del programa académico, seguido de las intrínsecas e identificando las extrínsecas como aquellas peor evaluadas.

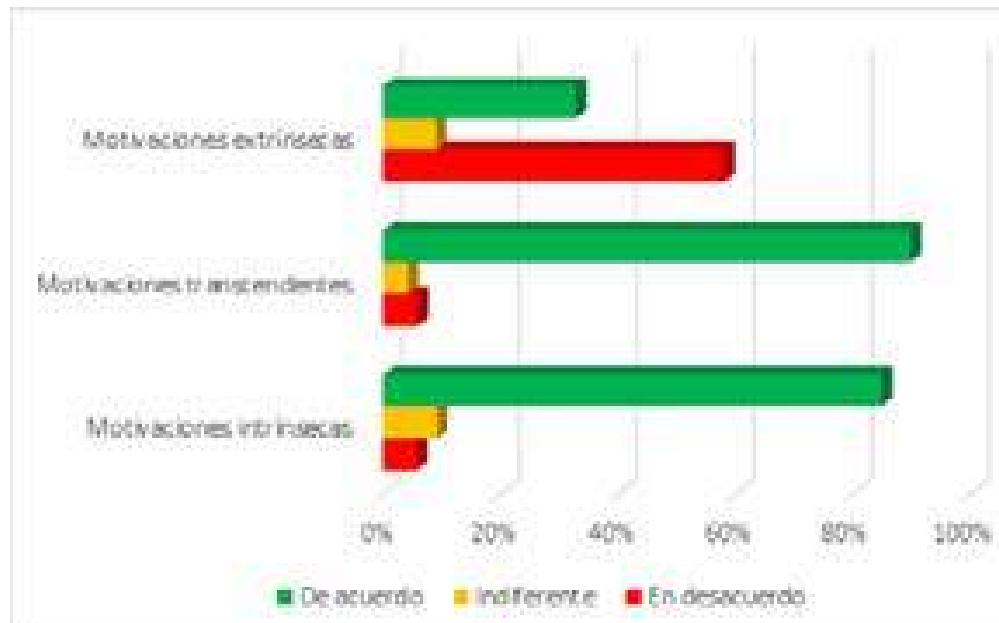

Figura 2. Comparativo de las motivaciones en opinión de los estudiantes del programa de 


\section{CONCLUSIONES}

Con respecto al género es importante señalar que las mujeres tienen una tendencia mayor a estudiar el programa de licenciatura en educación infantil que los hombres por tener mayor motivación y sensibilidad por la primera infancia.

Por otra parte, en opinión de los estudiantes matriculados en el programa académico de Licenciatura en Educación Infantil, los principales factores que han influido en la elección de este programa ha sido el impacto social que tiene el ejercicio docente en la construcción de una comunidad independientemente del contexto en que se desarrolle.

Reconocen la importancia de la profesión por lo que consideran que siempre hay oportunidades laborales a pesar de que los sueldos no resulten proporcionales al esfuerzo y dedicación dado que es una actividad que demanda de existencia de vocación de servicio y constante actualización para ofrecer un servicio de calidad.

A pesar de ser estudiantes que aun no han tenido contacto con el medio laboral, parecen tener una concepción arraigada de que el éxito del acto educativo se le ha atribuido solamente a los docentes y no se ha entendido como un compromiso de todos los actores tales como docentes, padres, estudiantes y sociedad en general.

\section{REFERENCIAS BIBLIOGRÁFICAS}

Álvarez-Gayou, J. (2011). Cómo hacer investigación cuantitativa. Fundamentos $y$ metodología. México: Paidós

Centurión, D. (2014). Preferencia de carreras entre estudiantes de la Universidad Americana. ACADEMO Revista de Investigación En Ciencias Sociales $Y$ Humanidades, 1(1), 55 - 68. Recuperado de http://revistacientifica. uamericana.edu.py/index.php/academo/article/ view/8

Bernal Guerrero, A., Jover Olmeda, G., Ruiz Corbella, M., \& Vera Vila, J. (2013). Liderazgo personal y construcción de la identidad profesional del docente. XXXII Seminario Interuniversitario de Teoría de la Educación. Liderazgo y educación (2013), p 17-42.

Day, C. (2006). Pasión por enseñar: la identidad personal y profesional del docente y sus valores. España: Narcea ediciones

Fuentealba Jara, R., \& Imbarack Dagach, P. (2014). Compromiso docente, una interpelación al sentido de la profesionalidad en tiempos de cambio. Estudios pedagógicos (Valdivia), 40(ESPECIAL), 257-273. Recuperado de https://scielo. conicyt.cl/scielo.php? pid=S0718$07052014000200015 \&$ script=sci_arttext\&tIng=e

Gámez, E., \& Marrero, H. (2003). metas y motivos en la elección de la carrera universitaria: un estudio comparativo entre Psicología, Derecho y Biología. Anales De Psicología, 19(1), 121-131. Recuperado de https://revistas.um.es/analesps/ article/view/27911

García-Quintero, C., \& Villamizar-Suárez, G. (2017). Análisis fenomenológico de la conciencia del docente a partir de sus prácticas evaluativas. Revista Perspectivas, 2(2), 49-59. https://doi. org/10.22463/25909215.1313

Gil-Madrona, P., Gómez-Barreto, I., \& GonzálezVíllora,S.(2016).Percepcióndelosestudiantes de maestro de educación infantil sobre su formación intercultural. MAGIS. Revista Internacional de Investigacion en Educacion, 9(18), 111128. Recuperado de https://www.redalyc.org/ pdf/2810/281049122007.pdf

Hansen, D. (2001). Llamados a enseñar. Barcelona: Idea Books. 
Kyriacou, C. y Coulthard, M. (2000). Undergraduates' views of teaching as a career choice. Journal of Education for Teaching, 26 (2), 117-127.

López-Jurado, M. y Gratacós, G. (2013). Elegir enseñar: propuesta del modelo antropológico de la motivación de Pérez López aplicada al ámbito de la educación. Estudios sobre educación, 24, 125-147.

Méndez- Omaña, J. P., \& Jaimes- Contreras, L. A. (2018). Clima social familiar e impacto en el rendimiento académico de los estudiantes. Revista Perspectivas, 3(1), 24-43. https://doi. org/10.22463/25909215.1422

Ryan, R. y Deci, E. (2000). La Teoría de la Autodeterminación y la facilitación de la motivación intrínseca, el desarrollo social y el bienestar. American Psychologist Association, 55 (1), 68-83. Recuperado de https://dadun. unav.edu/handle/10171/29567

Rodríguez-Muñiz, L. J., Areces, D., SuárezÁlvarez, J., Cueli, M. y Muñiz, J. (2019). ¿Qué motivos tienen los estudiantes de Bachillerato para elegir una carrera universitaria?. Journal of Psycholog

Said-Hung, Elias, Gratacós, Gloria, \& Cobos, Jorge Valencia. (2017). Factores que influyen en la elección de las carreras de pedagogía en Colombia. Educação e Pesquisa, 43(1), 31-48. https://dx.doi.org/10.1590/s15179702201701160978

Sánchez Lissen, Encarnación (2009). Dos caras de la carrera docente: satisfacción y desmotivación. Pedagogía Social. Revista Interuniversitaria, (16), 135-148. 1139-1723. Recuperado de: https://www.redalyc.org/ articulo.oa?id=1350/135012677010

Soza Ortiz, A. I., Talavera Hernández, F. J., \& Gutiérrez Salgado, N. M. (2017). Factores motivacionales que inciden en las $y$ los estudiantes para profesionalizarse en la carrera de Pedagogía con Mención en Educación Infantil en FAREM-Estelí en el II semestre del año 2016 (Doctoral dissertation, Universidad Nacional Autónoma de Nicaragua, Managua).

Troncoso Pantoja, C., Garay Lara, B., \& Sanhueza Muñoz, P. (2016). Percepción de las motivaciones en el ingreso a una carrera del área de la salud. Horizonte Médico (Lima), 16(1), 5561

Vidal Ledo, M., \& Fernández Oliva, B. (2009). Orientación vocacional. Educación Médica Superior, 23(2). Recuperado de http://scielo. sld.cu/scielo.php?script=sci_arttext\&pid =S0864-21412009000200011

Villamizar-Acevedo, G. A., Lozano-León, S. G., \& Sierra-Garavito, E. D. (2017). Creencias sobre las fuentes y formas de acceso al conocimiento generadas en las prácticas pedagógicas desde la perspectiva del estudiante. Revista Perspectivas, 2(1), 18-27. https://doi. org/10.22463/25909215.1281

Zapata, Beatriz Elena y Ceballos, Leonardo (2010). Opinión sobre el rol y perfil del educador para la primera infancia. Revista Latinoamericana de Ciencias Sociales, Niñez y Juventud, 8 (2), 1069-1082. Recuperado de: https://www. redalyc.org/articulo.oa?id=773/77315155021 